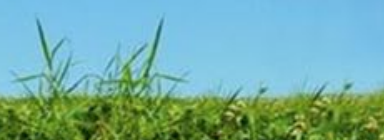

\title{
Adipositas
}

\section{Zu wenig Schlaf macht dick}

St-Onge MP, Roberts AL, Chen J et al. Am J Clin Nutr 2011;94(2):410-6.

Pilch W, Szyguła Z, Klimek AT et al. Int J Occup Med Environ Health 2010;23(2):167-74.

Das Lifestyle-Telegramm zu hochwertigen Publikationen mit dem Thema "Lebensstil" kann als Newsletter kostenlos abonniert werden und erscheint monatlich per Mail. Viele weitere entsprechende Kurzreferate gibt's unter: www.lifestyle-telegramm.de

Zhang Z, Hu G,

Caballero $B$ et al. Am J Clin Nutr 2011 93(6): 1212-9.
Kurze Schlafdauer führt offenbar dazu, dass die Energieund Fettaufnahme erhöht wird. Das würde den Zusammenhang zwischen wenig Schlaf und Fettleibigkeit erklären, der schon in mehreren Studien festgestellt wurde. 15 Männer und 15 Frauen im Alter von 30-49 Jahren mit einem BMI von $22-26\left(\mathrm{~kg} / \mathrm{m}^{2}\right)$, die normalerweise 7-9 Stunden pro Nacht schliefen, nahmen an der Crossover-Studie in einer New Yorker Klinik teil. Die Teilnehmer wurden jeweils fünf Nächte lang in randomisierter Reihenfolge unter verkürzten (4 Stunden/Nacht) bzw. normalen (9 Stunden/Nacht) Schlafbedingungen beobachtet. Am fünften Tag wurde jeweils die Nahrungsaufnahme festgehalten. Der Energieverbrauch wurde mit der „Doubly Labeled Water“-Methode aufgezeichnet.
Ergebnisse: Am fünften Tag der Kurzschlafphase nahmen die Teilnehmer mehr Energie auf (2813,6 \pm 593,0 kcal) als an Tag fünf der normalen Schlafphase (2517,7 $\pm 593,0 \mathrm{kcal} ; \mathrm{p}=0,023)$. Dieser Effekt in der Kurzschlafphase war in erster Linie auf einen erhöhten Konsum von Fetten $(20,7 \pm 37,4 \mathrm{~g} ; \mathrm{p}=0,01)$, und zwar besonders von gesättigten Fetten $(8,7 \pm 20,4 \mathrm{~g} ; \mathrm{p}=0,038)$ zurückzuführen. Die metabolische Rate im Ruhezustand (Kurzschlafphase: $1455,4 \pm 129,0 \mathrm{kcal} / \mathrm{d}$; Normalschlafphase: $1486,5 \pm 129,5 \mathrm{kcal} / \mathrm{d} ; \mathrm{p}=0,136)$ sowie der GesamtEnergieverbrauch (Kurzschlafphase: 2589,2 \pm 526,5 $\mathrm{kcal} / \mathrm{d}$; Normalschlafphase: $2611,1 \pm 529,0 \mathrm{kcal} / \mathrm{d}$; $\mathrm{p}=$ 0,832 ) unterschieden sich in den beiden Schlafphasen jedoch nicht signifikant.

\section{Wellness mit Nebenwirkung Regelmäßiges Saunieren bessert Lipidprofil}

Frauen, die regelmäßig in die Sauna gehen, können dadurch wohl ihr Lipidprofil verbessern. Das hat eine polnische Studie ergeben, an der 20 Frauen im Alter von 19 bis 21 Jahren teilgenommen haben. Sie nahmen sieben Mal jeweils im Abstand von zwei Tagen ein finnisches Saunabad von 30 oder 40 Minuten mit fünfminütiger Pause zum Abkühlen. BMI, Herzfrequenz und Blutdruck wurden vor und nach dem Saunieren gemessen. Während des Saunierens wurde rektal die Körpertemperatur überwacht. Vor jedem Saunabesuch sowie jeweils in den letzten beiden Minuten des Saunierens wurden die Sauerstoff-
Aufnahme und der $\mathrm{CO}_{2}$-Gehalt der ausgeatmeten Luft analysiert und der respiratorische Quotient errechnet. Blut wurde vor und nach den Saunagängen entnommen.

Ergebnisse: Die Körpertemperatur war beim letzten Saunabad niedriger als beim ersten. Plasmaverluste waren im siebten Saunagang größer als beim ersten. Der Lipid-Metabolismus steigerte sich nach jedem Saunabad. Nach wiederholten Saunagängen ließen sich ein reduzierter Gesamt- und LDL-Cholesterin-Spiegel sowie ein erhöhter HDL-Cholesterin-Spiegel verzeichnen.

\section{Inverse J-Kurve}

\section{Hypertonierisiko durch Kaffee}

Regelmäßiger Kaffeekonsum von $>3$ Tassen/Tag versus $<1$ Tasse/Tag ist wohl nicht mit erhöhtem Hypertonierisiko assoziiert. Jedoch scheint ein moderater Konsum von 1 bis 3 Tassen pro Tag das Risiko leicht zu erhöhen. Die Datenbanken MEDLINE, EMBASE, Agricola und Cochrane Library wurden bis einschl. 8/2009 durchsucht. Als geeignet galten prospektive Kohortenstudien, die sich mit der Assoziation zwischen Kaffeekonsum und Blutdruck bzw. neu aufgetretener Hypertonie befassten.

Ergebnisse: Aus sechs Studien wurden insgesamt 172567 Teilnehmer und 37135 neu aufgetretene Bluthochdruckfälle aufgenommen. Die Beobachtungszeit betrug im Mittel 6,4-33,0 Jahre. Im Vergleich zum nied- rigsten Kaffeekonsum [ $<1$ Tasse (ca. $237 \mathrm{ml}$ )/Tag] lag das gepoolte relative Risiko (RR) für Hypertonie in der nächsthöheren Kategorie (1-3 Tassen/Tag) bei 1,09 (95\%KI 1,01-1,18), in der zweithöchsten Kategorie (3-5 Tassen/Tag) bei 1,07 (95\%-KI 0,96-1,20) und in der höchsten Kategorie (> 5 Tassen/Tag) bei 1,08 (95\%-KI 0,96 $-1,21)$. Eine dosisabhängige Metaanalyse ergab eine inverse J-förmige Kurve mit einem steigenden Hypertonierisiko bei bis zu drei Tassen Kaffee pro Tag (RR bei 3 im Vergleich zu 0 Tassen Kaffee pro Tag: 1,07; 95\%-KI 0,97 bis 1,20) und einem abnehmenden bei höherem Konsum (RR bei 6 im Vergleich zu 0 Tassen Kaffee pro Tag: 0,99; 95\%-KI 0,89 bis 1,10). 